\title{
NUMERICAL CALCULATION OF SADDLE-POINT DISTRIBUTIONS IN INTERMEDIATE-ENERGY PROTON-HYDROGEN COLLISIONS
}

\author{
M. Horbatsch \\ Department of Physics and Astronomy York University, 4700 Keele Str., Toronto, Ontario M3J 1P3, Canada
}

(Received June 18, 1996)

\begin{abstract}
The time-dependent Schrödinger equation for the electronic motion in the field of two nuclei moving on classical trajectories is solved numerically by discretization using a Cartesian mesh. The symmetric proton-hydrogen collision system is considered for projectile velocities $v_{\mathrm{p}}$ comparable to the electron velocity in the initially occupied $\mathrm{H}(1 \mathrm{~s})$ ground state. For close collisions the dominant channels are captured to the projectile ground state and elastic scattering. The ionization process in this regime is dominantly populating electron continuum states with final momenta in the saddlepoint region, i.e., with small transverse momenta to the beam axis and longitudinal momentum values around $0.5 v_{\mathrm{p}}$. In this paper it is shown how to extract this information from large-scale numerical calculations.
\end{abstract}

Key words: atomic collisions, ionization, time-dependent Schrödinger equation, numerical solution.

PACS number(s): $34.50 \mathrm{~F}, 3470$

\section{INTRODUCTION}

The problem of ionization in ion-atom collisions in the non-perturbative regime has attracted a significant amount of attention in the experimental and theoretical atomic physics communities in the past 10 years. It turns out that even for the most fundamental collision systems such as protons on hydrogen $(\mathrm{p}-\mathrm{H})$, and antiprotons on hydrogen $(\overline{\mathrm{p}}-\mathrm{H})$ various calculations are in conflict with each other even for total ionization cross sections $[1,2]$. Of theoretical concern has been the question of how the non-perturbative ionization cross sections for the two systems approach the Born limit at high energies, which is independent of the projectile charge [3]. Experimentally the focus in recent years has been on the determination of electron emission characteristics [4-7]. This detailed information can shed more light on the ionization mechanism itself, but is also of practical concern for subjects such as radiation medicine with highly charged ions.

The experimental results in [4-7] have been controversial regarding the question whether a saddle-point mechanism exists at intermediate projectile velocities. To introduce this subject we provide a brief overview of some known ionization mechanisms.

It is well understood that at high energies, i.e., for projectile velocities much in excess of internal electron velocities for the given target bound state $\left(v_{\mathrm{p}}>>v_{\mathrm{e}}\right)$ the dominant ionization mechanism is direct target ionization, i.e., a single transition between a bound and final target continuum state. This process results in electrons emerging with relatively small velocities in the target rest frame.

In addition, there are other processes resulting in fast electrons. The binary-encounter mechanism can be pictured as a classical proton-electron collision likely to occur for impact parameters for which the projectile penetrates the charge cloud. This mechanism leads to very fast electrons emerging in the forward direction, and their signatures can be found in the proton deflections as well as in recoil atom momentum spectroscopy [8]. Electrons in the forward direction can emerge with velocities up to $2 v_{\mathrm{p}}$ due to this mechanism.

A third mechanism relevant at intermediate and slower projectile velocities, and particularly for higher projectile charges is capture to the continuum. In this case a transition occurs from a bound target state to a continuum state of the projectile. The electrons associated with this process have a small kinetic energy in the projectile frame. In the laboratory frame they show up as a characteristic cusp in the electron velocity distribution at near-zero transverse momentum and with a longitudinal velocity near $v_{\mathrm{p}}$ [9]. For slow asymmetric collisions (high projectile charge compared to an effective target charge of order unity) this becomes the dominant ionization mechanism.

For slow collisions $\left(v_{\mathrm{p}}<<v_{\mathrm{e}}\right)$ an adiabatic picture is appropriate, i.e., one can understand the physics in terms of energy correlation diagrams obtained from the diagonalization of the two-centre electronic Hamiltonian for fixed internuclear separation. The well-known LandauZener transition model of avoided level crossings and promotion of electronic orbitals populated at close internuclear separations to the continuum as the system separates, has been extended and applied successfully even to the antiproton-hydrogen system [10].

It has been shown recently that at such low velocities for which the quasimolecular picture is appropriate the overall low ionization probability is indeed dominated by a saddle-point mechanism, i.e., orbital promotion leads to ionized electrons moving with the saddle-point velocity $0.5 v_{\mathrm{p}}$ for the charge-symmetric system $\mathrm{p}-\mathrm{H}[11,12]$. This subject is presently attracting attention in the theoretical community [13].

For intermediate-energy collisions $\left(v_{\mathrm{p}} \approx v_{\mathrm{e}}\right)$ the importance of the saddle in the classical potential for the 


\section{HORBATSCH}

ionization process was claimed on the basis of classical trajectory calculations [14]. The model uses classical statistical mechanics to simulate quantum mechanics, and its validity can be questioned in this regime, since tunneling begins to play a role for the charge exchange mechanism. In particular, predictions were made that for charge-asymmetric systems with projectile charge $Z_{\mathrm{p}}$ and target charge $Z_{\mathrm{t}}$ the ionized electron distributions should be centered on the saddle-point velocity

$$
v_{\mathrm{s}}=\frac{v_{\mathrm{p}}}{1+\sqrt{Z_{\mathrm{p}} / Z_{\mathrm{t}}}}
$$

Several experimental attempts were made in recent years to address this question, but so far the situation remained unclear[4-7]. An interesting model that provides a reason for the importance of the classical saddle point has been suggested [15]: the rotation of the saddle with the internuclear axis can act as a Paul trap, i.e., stabilize an electronic wavepacket dynamically on the saddle.

A theoretical analysis of a one-dimensional model with soft-core Coulomb potentials solved numerically indicated that the classical potential saddle does not appear to play an important role for velocities $v_{\mathrm{p}} \geq v_{\mathrm{e}}[16]$. However, as is shown in the present study the situation changes in the realistic three-dimensional setting. Thus, it is quite possible that it lends support to the idea that the rotation of the saddle, i.e., Paul trapping is relevant for symmetric collisions.

In the last few years a new experimental technique for electron spectroscopy has been developed to resolve the controversy of saddle-point electrons at intermediate velocities $[17,18]$. Both studies consider the experimentally more accessible proton-helium system and single ionization only (one can assume that $Z_{\mathrm{t}} \approx 1.4$ ).

One investigation for $5-15 \mathrm{keV}$ proton impact is impact-parameter dependent (through a determination of the recoil $\mathrm{He}^{+}$momentum)[18] and clearly displays the complexity of the ionization process. For very close collisions at $v_{\mathrm{p}}=0.77 \mathrm{a}$.u. the electrons emerge predominantly as if the target atom explodes and little focusing of the electron wavepacket towards the projectile takes place. However, as the impact parameter increases, the electrons are broadly distributed over the saddle-point velocity with small but non-zero transverse momentum (in the $0.1-0.3$ a.u. range) towards the projectile. The ionized electrons are almost confined to the scattering plane.

The other investigation is not differential in impact parameter, but spans a wider velocity range $v_{\mathrm{p}}=0.6--2.4$ a.u. and provides also data for $\mathrm{C}^{6+}$ impact. It displays how the transverse momentum distribution broadens from being confined to the scattering plane for slow collisions to a half-width of 1.5 a.u. for the fast collision. For the longitudinal electron momentum distribution it demonstrates the importance of the saddle for proton velocities up to $v_{\mathrm{p}}=1.7 \mathrm{a}$.u. At $v_{\mathrm{p}}=2.4 \mathrm{a}$.u. the electron distribution is peaked at $v_{\mathrm{e}} \approx 0.5$ a.u., i.e., far below the saddle-point velocity. It is noteworthy that the transition appears to be quite abrupt. On the other hand the results for $\mathrm{C}^{6+}$ projectiles for impact velocities in the $v_{\mathrm{p}}=1.2-1.6$ a.u. range put in question the importance of the saddle point in charge-asymmetric systems: the emitted electron distributions display a cusp behaviour for the low velocity with a gradual shift to lower velocities, but well ahead of the saddle velocity as the projectile speed is increased. The classical trajectory results reported in [17] on the other hand appear to be dominated by the saddle point in the classical potential.

These findings clearly demonstrate the need of large-scale numerical solutions of the time-dependent Schrödinger equation (TDSE) in the impact parameter approximation. Basis-state expansion techniques have difficulties in dealing with the representation of the continuum. The two-centre problem alone when modeled to calculate predominantly bound-state populations suffers from a problem of overcompleteness when atomic orbitals on both nuclei are combined to form a basis. It has been recognized that states involving a third center (between the nuclei) need to be included to obtain reliable cross sections [19]. Nevertheless, it would be hard in these studies to analyze the continuum content of the wavefunction after the collision.

\section{THEORY}

The impact parameter approximation to the full three-body problem calls for the solution of the electronic TDSE for given internuclear trajectories $\mathbf{R}(t)$. The fixed trajectory serves as a source of energy for electronic excitations, including ionization. The traditional basis-state expansion technique to solve (in atomic units $\left.\hbar=m_{\mathrm{e}}=e=1\right)$

$$
i \frac{\partial}{\partial t} \psi(\mathbf{r}, t)=\left[-\frac{1}{2} \nabla^{2}-\frac{Z_{\mathrm{t}}}{r}-\frac{Z_{\mathrm{p}}}{|\mathbf{r}-\mathbf{R}(t)|}\right] \psi(\mathbf{r}, t)
$$

faces the following difficulties: the basis of eigenstates of the atomic Hamiltonian $H_{0}$ contains discrete and continuous states. Convergence in the latter states is very slow if one wishes to describe states travelling with the projectile or with the saddle-point velocity. Two-centre pseudobases, such as the Hylleraas basis can be used in quasimolecular adiabatic calculations and for very slow collisions in the TDSE to describe the charge transfer problem [20], but have difficulties at intermediate velocities due to the lack of translation factors. Two-centre atomic orbital bases need the addition of pseudostates to describe the continuum[2]. Converged calculations of this type represent a formidable computational task and still face the problem of how to extract the continuum information.

For the present study we have therefore adapted a numerical approach tested in the context of atomic ionization by superintense laser fields [21,22]. The method is described briefly with emphasis on the new aspects, namely how to extract continuum information from the propagated wavefunction. 
Space is discretized by a non-uniform mesh such that the region near the nuclei is resolved properly. At the same time we wish to span as much of space as possible to avoid artificial boundaries. This is achieved by a mapping of each Cartesian coordinate according to

$$
r_{i}=\beta_{i} \tan \left(\theta_{i}\right), \quad-\pi / 2<\theta_{i}<\pi / 2
$$

The parameters $\beta_{i}, i=1,2,3$ can be adjusted to determine at which scale in $r_{i}$ the mesh turns over from a fine to a coarse spacing, if an equidistant discretization in the new independent variables $\theta_{i}$ is introduced.

One of the advantages of the TD wavepacket approach over stationary scattering theory lies in the fact that the problem of the correct boundary conditions is always simple. Ionization can be described in terms of squareintegrable functions or on a mesh, as long as one terminates the propagation of the wavefunction before it hits the boundaries (that are also present in truncated square-integrable basis sets). The discretization of the TDSE in the $\theta_{i}$ variables permits, at least in principle, to impose the correct boundary condition, namely that $\psi(\mathbf{r}, t)$ vanishes for $r \rightarrow \infty$ with derivatives. Of course, we have to expect that the propagation is impeded once significant parts of the wavepacket reach regions where the mesh becomes coarse, i.e., for $\left|r_{i}\right|>\beta_{i}$.

The propagation of the wavefunction is straightforward after the Laplacian operator has been expressed in the $\theta_{i}$ variables. An alternating-direction implicit method that is unconditionally stable and can be implemented efficiently for modern computer workstations is used. The conservation of the norm of $\psi(\mathbf{r}, t)$ serves as one indicator that the time propagation is reasonable.

The wavefunction is monitored by the calculation of projections onto stationary target and travelling projectile eigenstates. The projectile is moving according to $\mathbf{R}(t)=\left(X_{0}+v_{\mathrm{p}} t, b, 0\right)$ where $b$ is the impact parameter and $X_{0}$ is chosen appropriately $\left(X_{0}=-Z_{\mathrm{p}} \times 10\right.$ a.u. $)$ to avoid sudden turn-on of excitations. We require

$$
a_{\mu}(t)=<\mu \mid \psi(t)>=\int d^{3} r \phi_{\mu}^{*}(\mathbf{r}) \psi(\mathbf{r}, t),
$$

and

$$
b_{\nu}(t)=<\nu, v_{\mathrm{p}} \mid \psi(t)>=\int d^{3} r \phi_{\nu}^{*}(\mathbf{r}) \mathrm{e}^{-i v_{\mathrm{p}} x} \psi(\mathbf{r}, t) .
$$

The projections are calculated, of course, as discrete sums. In principle, one has to determine the eigenfunctions $\phi_{\mu}$ on the mesh, since they do differ from the exact analytical eigenfunctions evaluated at the mesh points. While we use an iterative algorithm to determine an initial ground state in order to start the calculation without any excitations, for the purpose of bound-state projections we have used the exact hydrogenic eigenfunctions evaluated on the mesh.
In the limit of large times $\left(R(t)>Z_{\mathrm{p}} \times 10\right.$ a.u. $)$ the Hamiltonian goes over effectively into the atomic Hamiltonian (except for some Stark mixing between accidentally degenerate states). This means that the projections (2.3) and (2.4) stabilize and their moduli-squared become occupation probabilities for the bound target and projectile states.

If one is interested in target excitation and electron loss (due to ionization and charge transfer), one can calculate reasonably accurate transition probabilities using small meshes (e.g., $\beta_{i}=4$ a.u., and the number of points in the $(x, y, z)$ directions $64 \times 48 \times 48$ yields impact-parameter dependent probabilities accurate at the $1-2 \%$ level). These can be performed on a small workstation. However, to analyze the continuum content larger calculations are required. For the present work we chose $\beta_{x}=20$ a.u., $\beta_{y}=\beta_{z}=10$ a.u., and a size of $256 \times 128 \times 128$. This requires about $128 \mathrm{MB}$ of memory and typical jobs take several hours on a 64 -bit workstation (Sun Ultra-1 clocked at $143 \mathrm{MHz}$ ).

At a separation of $R(t) \approx 20$ a.u. after the collision we calculate a projected wavefunction from which the bound-state parts corresponding to the lowest three shells have been removed:

$$
\begin{aligned}
\mid \chi\left(t_{\mathrm{f}}\right)> & =\left|\psi\left(t_{\mathrm{f}}\right)>-\sum_{\mu=1}^{M} a_{\mu}\left(t_{\mathrm{f}}\right)\right| \phi_{\mu}> \\
& -\sum_{\nu=1}^{N} b_{\nu}\left(t_{\mathrm{f}}\right) \mid \phi_{\nu, v_{\mathrm{p}}}>.
\end{aligned}
$$

Note that the norm of the projected state $\mid \chi(t)>$ equals the overall ionization probability, while the total capture and target bound-state probabilities are given as the sums of the moduli squared of the coefficients calculated in (2.3) and (2.4).

In the final step the wavefunction $\chi\left(\mathbf{r}, t_{\mathrm{f}}\right)$ is Fourier analyzed to display its momentum content. The correct procedure would be to project it onto Coulomb eigenstates (for the two-centre problem). This represents a task, however, that appears to be prohibitive even on modern workstations, particularly since the states would have to be generated on the mesh. We have gained some confidence from the one-dimensional model work [16] (for which the Coulomb scattering states can be generated on the mesh) that the discrete Fourier transform of the projected wavefunction provides good results provided it is performed at distances of $R(t) \geq 20$ a.u.

To make use of fast Fourier transform techniques the three-dimensional wavefunction $\chi\left(\mathbf{r}, t_{\mathrm{f}}\right)$ is linearly interpolated to an equidistant mesh, and then transformed. The process of interpolation serves also as a measure of 'smoothness' of the original state. For intermediate and large impact parameters, for which the saddle-point region is prominently populated, there is practically no loss in norm in the interpolation process. 


\section{RESULTS}

Only a few examples are presented here to illustrate the transition regime from intermediate projectile velocities to fast collisions for the proton-hydrogen system. The results for the velocities of $v_{\mathrm{p}}=1,1.414$, and 2 a.u. correspond to impact energies of 25, 50, and 100 $\mathrm{keV}$ respectively. Due to the cylindrical geometry the total ionization cross section is dominated by intermediate
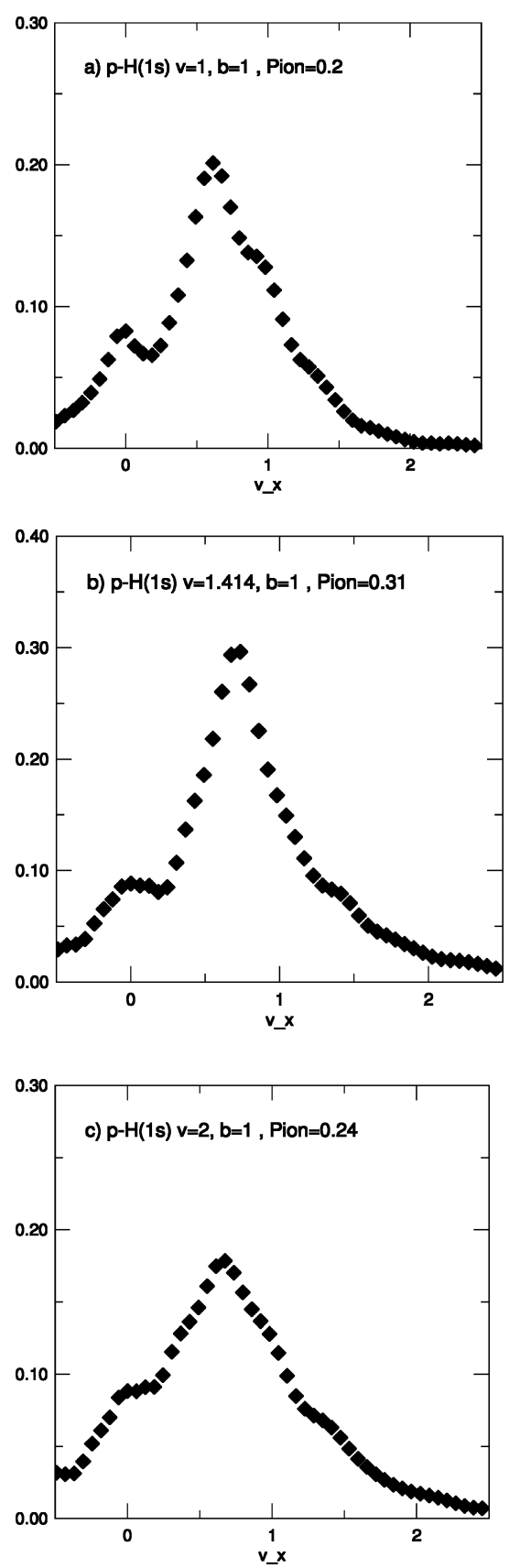

Fig. 1. Differential ionization probability $\frac{d P(b)}{d v_{x}}$ as a function of longitudinal electron velocity (momentum) for a fixed impact parameter of $b=1$ a.u. in proton-hydrogen collisions for different impact velocities: a) $v_{\mathrm{p}}=1$ a.u., b) $v_{\mathrm{p}}=1.414$ a.u., c) $v_{\mathrm{p}}=2$ a.u.


Fig. 2. Differential ionization probability $\frac{d P(b)}{d v_{x}}$ as a function of longitudinal electron velocity (momentum) for a fixed impact parameter of $b=2$ a.u. in proton-hydrogen collisions for different impact velocities: a) $v_{\mathrm{p}}=1$ a.u., b) $v_{\mathrm{p}}=1.414$ a.u., c) $v_{\mathrm{p}}=2$ a.u.

impact parameters, i.e., for given impact energy $E$ it is obtained from the ionization probabilities $P_{\text {ion }}(b)$ as

$$
\sigma_{\text {ion }}(E)=2 \pi \int_{0}^{\infty} P_{\text {ion }}(b) b d b
$$

Since we are showing differential $b$-dependent probabili- 
ties, it is useful to know that the cross sections for the systems considered are coming mostly from the $b=1 . .2$ a.u. range. Therefore, the data can be compared also to the experimental results which are summed over impact parameters [17].

Figs. 1a-c show the ionization probability for fixed impact parameter as a function of longitudinal electron momentum (velocity) along the beam axis. They clearly display that for the slower velocities the saddle-point region (in which the electron experiences very small accelerations) plays an important role. The data are in very good qualitative agreement with the experiment[17] as far as the position of the maximum probability is concerned. However, we note that the cusp structure near $v_{\mathrm{e}}=v_{\mathrm{p}}$ is more pronounced in the calculation (cf. cases $\mathrm{a}, \mathrm{b})$ than in the experiment, and that the experiment finds no enhancement in the electron signal at $v_{\mathrm{e}}=0$, i.e., no particular contribution from electrons emerging slowly from the target atom.

For the case of $v_{\mathrm{p}}=1 \mathrm{a} . \mathrm{u}$. there is a significant charge transfer probability of about $50 \%$, mostly to the ground state of the projectile. Projectile and target excitations contribute at much lower levels in accord with the known cross sections [2]. Given that the electron cloud experiences this dramatic motion (at lower energies it performs tunneling oscillations between both centres[20]), it is not too surprising that the ionization probability has three contributions corresponding to the saddle and to the two charge center velocities. What is rather surprising is the fact that the experimental data (for single ionization of helium by proton impact) are completely dominated by the saddle-point electrons with a small cusp feature. The latter can be amplified once a cut on the data is made to include only electrons with negligible transverse momenta.

We would like to stress that the importance of the saddle in the potential for the ionization mechanism is a function of impact parameter. For close collisions (e.g., $b=0.5$ a.u.) the distribution of the ionization probability over longitudinal electron momentum is quite irregular. This is in agreement with the experimental findings for $15 \mathrm{keV}$ proton-helium single ionization [18]. In this case a sizable fraction of electrons emerges in the backward direction (a double-scattering mechanism in which the projectile knocks the electron to collide with the target nucleus contributes to this channel), and the ionization probability is more reminiscent of the one for faster collisions, such as in fig. 1c.

Thus, one can argue that the saddle--point mechanism is most important for those electrons that are ionized while at a distance from the target nucleus. They are accelerated in the forward direction and the target nucleus has little influence to hold them back. It would be interesting to analyze the computational data further to understand at what internuclear distances the electrons obtain their final momenta in the forward direction. This question, however, is difficult to answer, as the use of plane-wave states for momentum analysis is not justified at small internuclear distances. A method has to be devised that takes the Coulomb potential into account.
One argument that can be found to explain the appearance of well-defined momentum values at which the electron distribution peaks is the phenomenon of spreading of wavepackets in coordinate space. As the projectile moves away from the target the potential in the region between the nuclei decreases in magnitude and has a small curvature. Thus, the ionized wavepacket delocalizes in coordinate space and narrows in momentum space around its mean value. This explanation does not rely on the precise position of the saddle point, i.e., holds also for the case of fast collisions. For the case of $v_{\mathrm{p}}=2 \mathrm{a} . \mathrm{u}$. a peak in the ionization probability occurs at $v_{\mathrm{e}} \approx 0.6$ a.u., roughly in accord with the $\mathrm{p}-\mathrm{He}$ experiment [17].

What is remarkable is that the mean momentum value for the ionized wavepacket is approximately independent of impact parameter (otherwise it would be washed out in the differential cross section given in [17]). It is possible that an analysis of the mean longitudinal momentum as a function of time (or internuclear distance) for various impact parameters will reveal interesting effects. Semiclassical Vlasov calculations for fast heavy ion collisions[23] have shown that the time evolution of the mean position (or velocity) of the electron distribution has a strong influence on the longitudinal motion of the recoils. As far as the ionized electronic wavepacket is concerned one might distinguish regimes where both nuclei are close together vs. post-collision effects.

A systematic investigation in which the projectile charge is varied is obviously called for. The comparison of experimental data for single ionization of He by singly and six-fold charged projectiles at similar velocities[17] suggests that the appearance of the peak in the electron longitudinal velocity distribution at the saddlepoint value for $\mathrm{p}-\mathrm{He}$ at intermediate velocities might be coincidental. It remains to be seen whether eq. (1.1) plays a role for some range of projectile charges and impact velocities.

Theoretical calculations for this problem in the present framework are in progress. We note that studies using the two-centre basis state expansion technique have found an unexpected importance of deeply bound projectile states for the calculation of ionization [24]. Such calculations are also of great interest to plasma physics.

\section{CONCLUSIONS}

In conclusion we would like to point out that the recent sophisticated experimental techniques to map out the wavefunctions (squared modulus) for ionized electron states in momentum space for fixed kinematic conditions [18] have spurred renewed theoretical interest in the problem. On the theoretical/computational side we are beginning to make progress, but there are still obstacles to be overcome. One of the difficulties when comparing the data quantitatively with experiment is that the calculation is performed in the scattering plane as fixed before the collision by a stationary target atom and the projectile beam axis. In the experiment the recoil ion momentum is used to define the plane, and thus, the the- 


\title{
M. HORBATSCH
}

oretical results need to be transformed. The transform is known, but is performed easily only for transition amplitudes known in closed form, e.g., from the Born approximation [25].

An unresolved issue in the present theoretical results is the appearance of sizable contributions of slow electrons emerging from the target atom, which are not seen in the recent experiments[17,18]. This is in spite of the fact that the experiments were designed to be very sensitive in this range. Further analysis of the computational data are required, i.e., it is possible that the removal of boundstate contributions is incomplete. It is also possible that ionized slow electrons (e.g., from field ionization of excited states by the receding projectile) are re-captured radiatively. This is a process not included in the present calculation based on the TDSE.

Once the validity of the numerical integration and
Fourier analysis technique is established various further applications can be envisaged. The extension to multiple ionization in highly-charged ion-atom collisions in the framework of a mean-field theory is one of them. Other interesting applications that are currently investigated in experiments lie in the field of ion-surface interactions.

\section{ACKNOWLEDGMENT}

I would like to thank Joachim Ullrich, Reinhard Dörner, and Lew Cocke for many discussions and for making the experimental data available prior to publication. The financial support of the Natural Sciences and Engineering Research Council of Canada is greatly appreciated.
[1] A. M. Ermolaev, Phys. Lett. A 149, 151 (1990).

[2] N. Toshima, Phys. Lett. A 175, 133 (1993).

[3] H. Knudsen, J. F. Reading, Phys. Repts. 212, 107 (1992).

[4] W. Meckbach et al., Phys. Rev. Lett. 57, 1587 (1986); J. Phys. B 24, 3763 (1991).

[5] T. J. Gay et al., J. Phys. B 23, L823 (1990).

[6] V. D. Irby et al., Phys. Rev. A 47, 2957 (1993).

[7] R. DuBois, Phys. Rev. A 48, 1123 (1993).

[8] R. Dörner, et al., Phys. Rev. A 45, 4572 (1992).

[9] R. G. Pregliasco, et. al., J. Phys. B 27, 1151 (1994).

[10] D. R. Schultz, et al., Phys. Rev. Lett. 76, 2882 (1996).

[11] M. Pieksma et al., Phys. Rev. Lett. 73, 46 (1994).

[12] M. Pieksma, S. Yu. Ovchinnikov, J. Phys. B 27, 4573 (1994).

[13] S. Yu. Ovchinnikov, J. H. Macek, Phys. Rev. Lett. 75,
2474 (1995).

[14] R. E. Olson, Phys. Rev. A 27, 1871 (1983); Phys. Rev. A 33, 4397 (1986).

[15] G. von Oppen, Europhys. Lett. 27, 279 (1994).

[16] M. Chassid, M. Horbatsch, J. Phys. B 28, L621 (1995).

[17] S. D. Kravis, et al., Phys. Rev. A 54, 1394 (1996).

[18] R. Dörner, et al., Phys. Rev. Lett. 77, 4520 (1996).

[19] T. G. Winter, Phys. Rev. A 37, 4656 (1988).

[20] H. J. Lüdde, R. M. Dreizler, J. Phys. B 14, 2191 (1981).

[21] M. Horbatsch, Phys. Rev. A 44, R5346 (1991).

[22] M. Horbatsch, J. Phys. B 24, 4919 (1991).

[23] M. Horbatsch, J. Phys. B 27, 2533 (1994).

[24] N. Toshima, J. Phys. B 27, L49 (1994).

[25] A. Salin, J. Phys. B 24, 3211 (1991).

\section{ОБЧИСЛЕННЯ РОЗПОДІЛІВ СІДЛОВОЇ ТОЧКИ ПРИ ПРОТОН-ВОДНЕВИХ ЗІТКНЕННЯХ ПРОМІЖНИХ ЕНЕРГІЙ}

\author{
М. Горбач \\ Факулътет фізики і астрономї, Університет Йорк, \\ 4700 Кіл стр., Торонто, Онтаріо МЗЈ 1Р3, Канада
}

\begin{abstract}
Здійснено числовий розв'язок залежного від часу рівняння Шредінгера для руху електрона у полі двох ядер, що рухаються вздовж класичних траєкторій, шляхом дискретизації на декартовій сітці. Симетрія протон-водневої системи при розсіянні розглядається для швидкостей бомбардуючого протона, порівняльних з швидкістю електрона у початково зайнятому основному стані $\mathrm{H}(\mathrm{SS})$. Для ближніх зіткнень домінуючими каналами є основний стан бомбардуючого протона і пружнє розсіяння. Іонізуючий процес у цьому режимі переважно заселюється станами електрона з неперервного спектра з кінцевим імпульсом в області сідлової точки, тобто з малим поперечним до осі пучка імпульсом і значенням поздовжнього імпульсу $0.5 v_{p}$. У цій статті показано, як отримати цю інформацію з великомасштабних числових розрахунків.
\end{abstract}

